\title{
Consumo de drogas en estudiantes universitarios de primer curso
}

\section{Addictive substance use among first-year university students}

\author{
Adriana Jiménez-Muro Franco*; Asunción Beamonte \\ San Agustín ${ }^{\star *}$; Adriana Marqueta Baile ${ }^{\star}$; PIllar \\ Gargallo Valero**; Isabel Nerín de la Puerta*
}

* Departamento de Medicina y Psiquiatría. Unidad de Tabaquismo FMZ. Facultad de Medicina, Universidad de Zaragoza.

** Departamento de Métodos Estadísticos. Escuela Universitaria de Estudios Empresariales, Universidad de Zaragoza.

Enviar correspondencia a:

Adriana Jiménez-Muro Franco. Unidad de Tabaquismo FMZ.

Facultad de Medicina. Universidad de Zaragoza

C/ Domingo Miral s/n, Edif. A, $1^{a}$ planta. 50009 Zaragoza

e-mail: adrijmf@unizar.es

\section{RESUMEN}

Objetivo: Mejorar el conocimiento de algunos de los factores y actitudes que pueden influir en el consumo de drogas de los estudiantes universitarios españoles de primer curso, en especial en lo referente al tabaco. Métodos: Estudio descriptivo mediante cuestionario anónimo y voluntario. Variables de estudio: sexo, edad, entorno familiar, consumo de tabaco, alcohol y cannabis, actitudes a favor y en contra de fumar, percepción de peligrosidad y percepción de las conductas de los amigos. Resultados: 2445 alumnos, 1014 hombres (42\%) y 1431 mujeres (58\%), edad media 19 años. En la población estudiada un mayor porcentaje de mujeres que de hombres afirma que fumar tabaco es apetecible, creen que les ayuda a estar delgadas y les hace sentir bien; los hombres afirman en mayor proporción que facilita la relación grupal. Los universitarios que fuman tabaco se iniciaron en el consumo de alcohol antes que los que no fuman. El 16,5\% del total de la muestra consume cannabis a diario, ocasionalmente o los fines de semana. El 10,4\% afirma que sus amigos han probado y consumen otras drogas. Conclusiones: En este estudio las mujeres y los hombres tienen distinta percepción sobre el tabaco, y el porcentaje de mujeres fumadoras es mayor. El nivel de tolerancia hacia el alcohol contribuye a que los jóvenes tengan una menor percepción del riesgo. Se podria pensar que el inicio tabáquico temprano puede actuar como la puerta de entrada para el consumo de alcohol y otras drogas.

Palabras clave: tabaquismo, estudiantes universitarios, alcohol, cannabis, drogas, actitudes.

\section{ABSTRACT}

Objective:To analyze several factors and attitudes related to drug use among first-year Spanish university students, with special reference to tobacco. Methods: Descriptive study using voluntary and anonymous questionnaire. Study variables: sex, age, family environment, tobacco, cannabis and alcohol use, attitudes in favour of or against smoking, perceived danger of drugs and perceptions of friends' behaviour. Results: 2445 students, 1014 (42\%) men and 1431 (58\%) women, mean age 19 years. Women in this study see smoking as appealing, believe it helps them stay slim and claim that it makes them feel good. Of the total sample (men and women), 16.5\% use cannabis daily, occasionally or at weekends, while $10.4 \%$ state that their friends have tried or use other drugs. Conclusions: In this sample women and men have different perceptions about tobacco. Level of tolerance and permissiveness with regard to alcohol contributes to the fact that young people perceive less risk in relation smoking. Starting smoking tobacco early may make the use of alcohol and other drugs more likely.

Key words: smoking, university students, alcohol, cannabis, drugs, attitudes. 


\section{INTRODUCCIÓN}

L a experimentación y el inicio de consumo de tabaco, alcohol y otras drogas ilegales se produce típicamente en los primeros años de la adolescencia'. Referente al consumo de tabaco, las encuestas realizadas en los años 2003 y 2006 en jóvenes españoles muestran una disminución del $4 \%$ en los fumadores a diario para el rango de edad de 16 a 24 años, pero aún así la prevalencia, del 28,2\%, sigue siendo alta ${ }^{2}$. Independientemente de esta disminución en el porcentaje de fumadores, diversos estudios apuntan una menor edad de inicio en el consumo, siendo actualmente en España los 13,4 años en escolares de 14 a 18 años ${ }^{3}$. Diferentes encuestas realizadas en nuestro país reflejan que el tabaco sigue siendo la primera sustancia de experimentación en los adolescentes. El inicio al consumo de alcohol se produce a edades muy tempranas, en la mayoría precedido del consumo experimental de tabaco. Se ha observado en los últimos años un cambio en el patrón de consumo de alcohol de los jóvenes, en el que a pesar de disminuir el consumo a diario, aparece más intenso durante el fin de semana y asociado con el consumo de otras sustancias adictivas'. El consumo de alcohol ha pasado a ser un componente esencial del tiempo de ocio de los jóvenes. La importante tolerancia social existente y la escasa percepción del riesgo asociado a la ingesta de bebidas alcohólicas han contribuido a la generalización del consumo y a una normalización de esta conducta ${ }^{4}$. Según los datos de la Encuesta Nacional de Salud (ENS) de España de 2006 el 76\% de los jóvenes entre 16 y 24 años afirman que han bebido alcohol en los últimos 12 meses $^{2}$.

Entre los factores que influyen en el inicio del consumo de alcohol y de tabaco se relacionan aspectos familiares, socioeconómicos, cognitivos y emocionales ${ }^{5}$, siendo importante en el caso de este último la presencia de tabaquismo en el entorno, tanto familiar como entre los amigos, así como la presión social y las actitudes a favor o en contra del tabaco. La Teoría del Aprendizaje Social contempla los efectos de las redes de asociación, de manera que aquellas personas con las cuales se interactúa habitualmente, delimitan patrones de conducta que al ser observados de manera repetida tienen más probabilidades de ser aprendidos ${ }^{6}$. Así en la mayoría de los modelos de inicio del consumo de tabaco se incluye tener familiares e iguales fumadores como un factor predictor? Diversos estudios confirman que unos padres que fumen "abren la puerta" al consumo de tabaco de sus hijos ${ }^{8}$. También se ha descrito la asociación entre el número de fumadores existentes que hay en el hogar y el inicio en el consumo ${ }^{9}$. Por otra parte, se han identificado además otros condicionantes cognitivos que podrían contribuir a la consolidación de la conducta de fumar entre los jóvenes, como una mayor seguridad y confianza y mayor facilidad para relacionarse; en este sentido, los jóvenes utilizarian el cigarrillo como un recurso para interactuar con sus iguales'. Aparte perciben como positivos algunos efectos relacionados directamente con la ingesta de nicotina, como la sensación de placer o en el caso de las jóvenes, la percepción de que fumar les ayuda a controlar el peso $0^{10,11,12}$. Por el contrario, existen factores protectores que ayudarán a mantener la determinación de no fumar, como puede ser la realización de ejercicio físico y un nivel educacional más alto ${ }^{13}$ 14. En este sentido, se ha descrito una relación entre el nivel de estudios y tabaquismo, con un porcentaje menor de fumadores entre universitarios que entre los jóvenes que trabajan ${ }^{15}$, siendo aquellos con el nivel de estudios más alto (doctorado) los que presentan una menor prevalencia ${ }^{16}$. Sin embargo, un porcentaje de alumnos que acceden a estudios superiores inicia o consolida la conducta de fumar a lo largo de su etapa universitaria. Por todo ello, un mejor conocimiento de los factores condicionantes puede contribuir a mejorar las actuaciones preventivas dirigidas a este colectivo de jóvenes. El riesgo percibido ante distintas conductas de consumo de drogas (medido por la proporción de personas que piensa que una determinada conducta puede provocar bastantes o muchos problemas) puede ser un indicador indirecto de la evolución del consumo. El consumo de drogas legales podría facilitar el posterior consumo de cannabis, considerada primera droga ilegal consumida por los jóvenes españoles y la que se consume a edad media más temprana. En los últimos años ha disminuido claramente la percepción del riesgo ante el consumo de alcohol, cannabis y el consumo esporádico de cocaína, especialmente para el cannabis; entre los jóvenes de 14 a 18 años la proporción de los que pensaban que esta droga podía producir muchos o bastantes problemas pasó de 60\% en 1994 a 35\% en $2005^{17}$. Aun así la percepción de riesgo del consumo habitual de cannabis es algo superior al atribuido al consumo diario de tabaco e inferior al atribuido al consumo habitual de otras drogas ilegales ("pastillas", cocaína y heroína) ${ }^{3}$.

El consumo de drogas entre quienes se están desarrollando física y socialmente constituye un gran problema a nivel mundial. Por ello es importante conocer las pautas de consumo de drogas legales e ilegales en España para poder realizar un abordaje adecuado a las necesidades a nivel nacional así como europeo ${ }^{17}$.

El objetivo de este estudio es mejorar el conocimiento de algunos de los factores y actitudes que pueden influir en el consumo de drogas de los estudiantes universitarios españoles de primer curso, en especial en lo referente al tabaco.

\section{MÉTODO}

Se realizó un estudio transversal descriptivo. La población objetivo fueron los alumnos que ingresaban por primera vez en la Universidad de Zaragoza en el curso 2005/2006, que incluye los centros de Zaragoza, Huesca y Teruel. El proyecto se planteó con el visto bueno del Vicerrectorado de Estudiantes de la Universidad, que solicitó mediante un escrito formal la colaboración a los Decanos y Administradores de todos los centros. 


\section{Sujetos}

El número total de matriculados de nuevo ingreso en primer curso en la Universidad de Zaragoza fue de 6014 alumnos. Actualmente se puede realizar la matrícula de forma presencial o por Internet; en este estudio sólo se pudo acceder a los alumnos que la realizaban de forma presencial, ya que por parte del Vicerrectorado de Alumnos no se autorizó incorporar el cuestionario a la aplicación de Internet existente para la matrícula.

Se recogieron 2662 cuestionarios, que suponía un 44,5\% del total de los alumnos matriculados en primer curso. Se excluyeron del estudio aquellos estudiantes mayores de 25 años, por considerar que sus condicionantes eran distintos. $Y$ también fueron excluidos del análisis aquellos cuestionarios a los que les faltaba al menos una pregunta por contestar. Por lo tanto, la muestra final analizada fue de 2445 alumnos, 1014 hombres $(41,5 \%)$ y 1431 mujeres $(58,5 \%)$ con edad media de 19 años $( \pm 1,6)$.

\section{Instrumento y recogida de datos}

Se diseñó un cuestionario de 29 ítems basado en encuestas sobre consumo de drogas realizadas en el medio escolar y en el Programa Europeo de Prevención del Tabaquismo en adolescentes, proyecto ESFA (European Smoking Prevention Framework Approach) ${ }^{18,19}$, con preguntas cerradas sobre el consumo de tabaco, además de las actitudes y creencias relacionadas con esta sustancia ${ }^{10}$, 20, 21. Además se incluyeron preguntas sobre consumo de alcohol y cannabis, sobre riesgo percibido y peligrosidad de éstas y de las demás drogas. Al elaborar el cuestionario, se introdujeron los términos "borrachera" para hacer referencia a los episodios de intoxicaciones etílicas, "porros" para definir el cigarrillo con hachís o marihuana, y "pastillas" para las cápsulas o comprimidos que en su composición llevan sustancias tales como anfetaminas y/o éxtasis.

Los alumnos lo cumplimentaron durante el periodo de matrícula de forma voluntaria y anónima. Para garantizar el anonimato, cada alumno introducía el cuestionario en un sobre sin nombre y lo cerraba antes de entregarlo a los responsables de secretaría. Una vez finalizado el periodo de matriculación, los cuestionarios fueron recogidos por los propios investigadores en las distintas facultades y escuelas universitarias, para asegurar la correcta clasificación de los centros.

El consumo de tabaco se recogió con una pregunta cerrada con 4 opciones de respuesta: fumador a diario, fumador ocasional, ex fumador y no fumador. Los ítems del cuestionario que recogen las actitudes relacionadas con el tabaco se agruparon en dos niveles ${ }^{5}$, con posibilidad de respuesta dicotómica para cada una de ellas: actitudes a favor de fumar ("ayuda a estar delgado", "hace que uno esté más relajado", "hace más fácil estar en grupo", "es apetecible", "da más seguridad y/o confianza", "te hace sentir bien") y actitudes en contra de fumar ("es desagradable", "es perjudicial", "está muy mal visto", "el tabaquismo pasivo es perjudicial para la salud"). Para analizar las variables del entorno se preguntó acerca de la situación del padre y de la madre en relación con el tabaco (fuma cada día, ocasionalmente, ex fumador/a, no ha fumado nunca). Se valoró la percepción que los futuros universitarios tienen acerca del porcentaje de fumadores entre las personas de su edad y la población general, con 3 opciones de respuesta (la minoría, la mitad, la mayoría) y la intención personal de fumar en el futuro. Además se preguntó acerca del consumo propio de alcohol, en qué lugares lo consumen y con qué frecuencia en los últimos 6 meses. Seguidamente para analizar el consumo de cannabis se introdujo la pregunta: "¿Actualmente fumas porros?" con 5 posibilidades de respuesta. Se recogió la percepción de peligrosidad sobre el tabaco y otras drogas, valorada con una escala tipo Likert de 1 (nada peligrosa para tu salud) a 5 (muy peligrosa para tu salud). Se incluyeron preguntas sobre la práctica de ejercicio físico y la disponibilidad económica en relación con tener o no un trabajo remunerado.

\section{Análisis de datos}

Con la información proporcionada por los cuestionarios se elaboró una base de datos en SPSS $14.0^{\circ}$. El estudio estadístico se inició con un análisis exploratorio tanto numérico como gráfico de los datos. Posteriormente, se llevaron a cabo análisis inferenciales que permitieron determinar relaciones entre variables cualitativas por medio de tablas de contingencia y contrastes Chi cuadrado de Pearson. Para analizar la dependencia entre variables cuantitativas y cualitativas se utilizaron Test de diferencia de medias. El nivel de significación considerado estadisticamente significativo fue $p<0,05$. Se realizó una regresión lineal para establecer si existía correlación entre la edad de inicio de consumo de tabaco y la edad de inicio de consumo de alcohol representado por un diagrama de dispersión.

\section{RESULTADOS}

Del total de la muestra, el 17,3\% afirma fumar tabaco a diario y $13,5 \%$ ocasionalmente. La edad media de inicio en el consumo de esta población era 14 años $( \pm 2,27)$. La prevalencia de tabaquismo es mayor entre las mujeres $(33 \%)$ que en los varones (27\%) sin existir diferencias por sexo en el $n^{\circ}$ de cigarrillos/dia, de media $12( \pm 5,76)$. El 67\% fuma su primer cigarrillo en la primera hora después de levantarse. Al 79\% le gustaría dejar de fumar y de éstos, el $55 \%$ dice haber realizado algún intento serio de abandono, con 2,21 $( \pm 1,36)$ intentos de media.

Para estudiar las diferentes actitudes entre fumadores y no fumadores de esta muestra de alumnos se agruparon las categorias de fumador a diario y ocasional (fumador) y no ha fumado nunca y ex fumador (no fumador). Se encontraron diferencias significativas en los ítems correspondientes a las actitudes a favor de fumar: los alumnos que son 
fumadores consideran en mayor medida que el tabaco no es desagradable, dicen que les ayuda a relajarse, es apetecible, les da más seguridad y/o confianza, les hace sentir bien y creen que les ayuda a estar delgados/as, aunque consideran en mayor proporción que "fumar está muy mal visto" con significación estadística $(p<0,001)$; por el contrario, en esta muestra aquellos alumnos que no son fumadores creen en mayor proporción que fumar es desagradable $(87,9 \%$ frente a $40,8 \%)$ y que el tabaquismo pasivo es perjudicial $(69,9 \%$ frente a 30,1\%) (ver tabla 1).

El 97\% del total de los estudiantes encuestados cree que inhalar aire contaminado por humo de tabaco (tabaquismo involuntario) es perjudicial para la salud, pero al analizar este ítem entre los no fumadores y aquellos que fuman, son éstos los que piensan en mayor proporción que no es perjudicial, con una fuerte significación estadística $(p<0,0001)$.

Al analizar las actitudes frente al consumo de tabaco por sexo, se observó que una mayor proporción de mujeres que de hombres de este estudio lo perciben como apetecible $(p<0,05)$, creen que les ayuda a estar delgadas $(p<0,01)$ y les hace sentir bien $(p<0,05)$, mientras que un porcentaje mayor de hombres que de mujeres considera que facilita la relación en grupo con sus amigos $(p<0,003)$ (ver tabla 2).

Respecto a las variables que hacen referencia al tabaquismo de los padres, el 35\% de los alumnos analizados dice que su padre es fumador a diario u ocasional, y el $30 \%$ afirma lo mismo de la madre. En esta muestra encontramos que, en los alumnos que son fumadores en relación con los que no lo son, existe una mayor asociación con que sus padres sean fumadores.

La conducta de la madre con respecto al tabaco se asocia con el hecho de que los hijos fumen reflejado en las categorias de fumadora a diario y ocasional $(p<0,003)$. En el caso del padre, esta asociación es únicamente en la categoría de fumador a diario $(p<0,001)$.

En cuanto a la percepción que los alumnos de este estudio tienen sobre la proporción de personas fumadoras entre la población general, el $41 \%$ contestó que fuma la mayoría, y con respecto a las personas de su edad el 51\% percibe lo mismo. En este último aspecto, el $82 \%$ de los alumnos que fuman ante la pregunta "¿cuántos de tus amigos fuman cigarrillos?", dicen que la mayoría o todos, por el contrario, el 78\% de los no fumadores dicen que la mitad o la minoría. Al analizar la intención de fumar en el futuro en el total de la muestra, el 16\% cree que fumará, mientras que el $22 \%$ no lo sabe. Existen diferencias por sexo ya que en este estudio hay más hombres que dicen que no fumarán mientras que una proporción mayor de mujeres que de hombres creen que seguro que sí seguirán fumando $(p<0,001)$.

La edad de inicio al consumo de alcohol es de 15,10 años $( \pm 1,53)$ sin existir diferencias por sexo. El $66,2 \%$ del total de la muestra se ha emborrachado alguna vez en su vida. En la tabla 3 se muestran las diferencias entre hombres y mujeres en el número de veces que se han emborrachado en los últimos 6 meses. Es importante señalar que el 8,2\% de hombres dice haberse emborrachado más de 12 veces en los últimos 6 meses. Los hombres se han emborrachado con mayor frecuencia en los últimos 6 meses $(p<0,001)$. En estos alumnos hay que destacar que los hombres beben alcohol más frecuentemente entre semana (los dias de clase), mientras que las mujeres dicen hacerlo en fin de semana, $(p<0,001)$. No se encontraron diferencias por sexo en el lugar donde se consume alcohol. Si se han encontrado diferencias respecto al alcohol entre aquellos estudiantes que fuman y los que no fuman tabaco. En este estudio los estudiantes fumadores probaron por primera vez el alcohol con una media de edad de 14,56 años $( \pm 1,55)$, mientras que aquellos que no fuman lo hicieron a los 15,37 años $( \pm 1,43)$ $(p<0,001)$. Los fumadores dicen haberse emborrachado al menos una vez en su vida en mayor proporción que los no fumadores $(p<0,001)$ y también lo han hecho con mayor frecuencia en los últimos 6 meses $(p<0,001)$. Al realizar una regresión lineal entre la edad de inicio del consumo de tabaco y la edad de inicio de consumo de alcohol, el coeficiente de correlación entre estas dos variables fue de $r$ $=0,399$, lo que muestra una correlación positiva moderada entre ambas. Esto quiere decir que los que empezaron a fumar antes de los 15 años en general también se iniciaron precozmente en el alcohol, mientras que los que empezaron a fumar después de los 15 años se iniciaron más tardiamente en el alcohol (Figura 1).

El $16,5 \%$ del total de la muestra dice que fuma cannabis (a diario, ocasionalmente o los fines de semana). De éstos, el 57\% afirma que sus amigos también lo hacen. El 32,8\% de los consumidores de cannabis creen que su consumo no provoca adicción frente al $67,2 \%$ de los que no consume que consideran que si $(p<0,001)$. Existen también diferencias en cuanto a la percepción de peligrosidad, el $15,8 \%$ de los que consumen cannabis frente al $84,2 \%$ de los que no consumen considera que no es peligroso para su salud $(p<0,001)$.

En la tabla 4 se detallan las diferencias por sexo. Los hombres que dicen fumar porros afirman hacerlo a diario en mayor proporción que las mujeres $(p=0,001) y$, por otra parte, el porcentaje de mujeres que nunca los han probado es superior al de hombres $(p=0,001)$.

El 5,6\% del total de la muestra se define como ex fumador de tabaco, pero de éstos el 22,7\% fuma porros ocasionalmente, $2,3 \%$ los fines de semana y $3,8 \%$ a diario. El 84\% de los fumadores de tabaco a diario de este estudio han probado el cannabis en alguna ocasión, y de éstos, el $12,3 \%$ lo fuma a diario también. De aquellos estudiantes que no han fumado nunca tabaco un 5\% afirma fumar cannabis ocasionalmente.

El 10,4\% del total de la muestra afirma que sus amigos consumen otras sustancias además de las ya referidas, como son cocaína y "pastillas". En la escala de peligrosidad de drogas del 1 al 5, se puntuó de media, en todas ellas, por encima de 3, siendo el alcohol la sustancia considerada como menos peligrosa. Aquellos alumnos que fuman tabaco y cannabis tienen menor percepción de riesgo ante el consumo de otras sustancias, con diferencias estadísticamente significativas en todas ellas: alcohol 
$(p<0,003)$, "pastillas", cocaína $(p<0,002)$, y heroína $(p<0,001)$.

Del total de la muestra, el 36\% realiza ejercicio físico regularmente y el $16 \%$ compatibiliza sus estudios con algún tipo de trabajo remunerado. Al analizar estas variables según el consumo de tabaco, se observó que los estudiantes que fuman hacen menos deporte $(p<0,001)$ y compatibilizan sus estudios con trabajo remunerado en mayor proporción $(p<0,001)$. Aquellos estudiantes que fuman porros (a diario, ocasionalmente o los fines de semana) compatibilizan en mayor proporción que el resto sus estudios con trabajo remunerado $(p<0,001)$, pero no se encontraron diferencias respecto al ejercicio físico.

Tabla 1. Diferencias entre fumadores $(F)$ y no fumadores (NF) en las actitudes ante el tabaco

\begin{tabular}{|l|c|c|c|}
\hline Actitudes & NF (\%) & F (\%) & p \\
\hline Ayuda a estar delgado/a & 11,5 & 16,1 & 0,002 \\
\hline Relaja & 27,4 & 57 & 0,001 \\
\hline Facilita relación grupal & 17,4 & 12,5 & 0,002 \\
\hline Es apetecible & 12,1 & 70,9 & 0,001 \\
\hline Da seguridad y/o confianza & 8,7 & 12,9 & 0,001 \\
\hline Te hace sentir bien & 7,3 & 37,9 & 0,001 \\
\hline Es desagradable & 87,9 & 40,8 & 0,001 \\
\hline Es perjudicial & 99 & 98,5 & 0,26 \\
\hline Está muy mal visto & 27,6 & 40,8 & 0,001 \\
\hline Tabaquismo pasivo perjudicial & 69,9 & 30,1 & 0,001 \\
\hline
\end{tabular}

*se consideran diferencias estadisticamente significativas valores $p \leq 0,05$

Tabla 2. Diferencias por sexo en las actitudes ante el tabaco

\begin{tabular}{|l|c|c|c|}
\hline Actitudes & Hombres (\%) & Mujeres (\%) & $\mathbf{p}$ \\
\hline Ayuda a estar delgado/a & 11,5 & 14,1 & 0,006 \\
\hline Relaja & 36,1 & 37 & 0,05 \\
\hline Facilita relación grupal & 18,5 & 14 & 0,003 \\
\hline Es apetecible & 28,1 & 31,8 & 0,05 \\
\hline Da seguridad y/o confianza & 10,5 & 9,6 & 0,05 \\
\hline Te hace sentir bien & 14,8 & 17,9 & 0,04 \\
\hline Es desagradable & 74,1 & 73,2 & 0,05 \\
\hline Es perjudicial & 98,7 & 99,1 & 0,38 \\
\hline Está muy mal visto & 32,5 & 30,5 & 0,05 \\
\hline Tabaquismo pasivo perjudicial & 40,9 & 51,9 & 0,001 \\
\hline
\end{tabular}

*se consideran diferencias estadisticamente significativas valores $p \leq 0,05$
Tabla 3. Diferencias por sexo en el número de "borracheras" en los últimos 6 meses (valores absolutos)

\begin{tabular}{|l|l|l|l|}
\hline Frecuencia & \multicolumn{1}{|c|}{ Hombres } & \multicolumn{1}{|c|}{ Mujeres } & \multicolumn{1}{|c|}{ p } \\
\hline Ninguna vez & $44,7 \%(468)$ & $51,8 \%(756)$ & 0,001 \\
\hline De 1 a 6 veces & $33,9 \%(335)$ & $35,7 \%(500)$ & 0,001 \\
\hline De 6 a 12 veces & $8,2 \%(81)$ & $6,9 \%(96)$ & 0,001 \\
\hline De 12 a 24 veces & $8,2 \%(81)$ & $4,1 \%(58)$ & 0,001 \\
\hline Más de 24 veces & $5 \%(49)$ & $1,5 \%(21)$ & 0,001 \\
\hline TOTAL & $100 \%(1014)$ & $100 \%(1431)$ & \\
\hline
\end{tabular}

*se consideran diferencias estadisticamente significativas valores $p \leq 0,05$

Tabla 4. Diferencias por sexo en el perfil de consumo de cannabis (valores absolutos)

\begin{tabular}{|l|c|c|c|}
\hline Consumo de cannabis & Hombres & Mujeres & $p$ \\
\hline Nunca ha consumido & $47,6 \%(493)$ & $49,3 \%(714)$ & 0,001 \\
\hline No consume pero ha probado & $33,2 \%(330)$ & $36,2 \%(512)$ & 0,001 \\
\hline Consume ocasionalmente & $11,7 \%(116)$ & $10,5 \%(149)$ & 0,001 \\
\hline Consume los fines de semana & $2,3 \%(23)$ & $2,1 \%(30)$ & 0,001 \\
\hline Consume a diario & $5,2 \%(52)$ & $1,8 \%(26)$ & 0,001 \\
\hline TOTAL & $100 \%(1014)$ & $100 \%(1431)$ & \\
\hline
\end{tabular}

*se consideran diferencias estadisticamente significativas valores $p \leq 0,05$

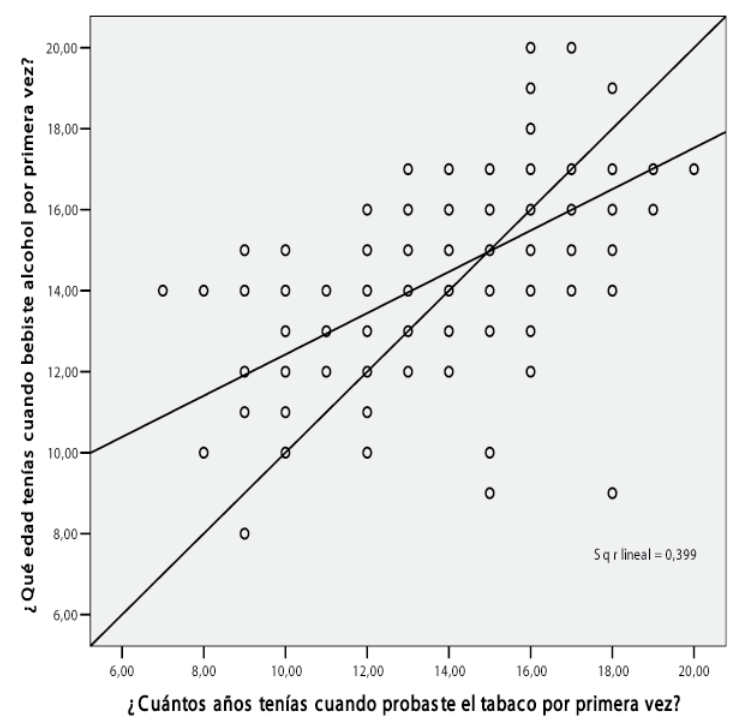

Figura 1. Regresión lineal entre la edad de inicio en el tabaco y la edad de inicio en el alcohol 


\section{DISCUSIÓN}

Los resultados de nuestro estudio ponen de manifiesto que los estudiantes analizados que son fumadores muestran en general actitudes claramente favorables al consumo de tabaco, lo cual se ha descrito como uno de los factores predictivos más potentes para consolidar la conducta de fumador ${ }^{22}$. Por el contrario, estos mismos alumnos fumadores consideran que fumar está muy mal visto, lo que indica el cambio en la aceptación social que se está dando en nuestra sociedad en los últimos años. Respecto al tabaquismo involuntario, aunque la mayoría de los encuestados lo considera perjudicial para la salud, son también los estudiantes que fuman los que muestran menor acuerdo con esta afirmación.

Los estudiantes universitarios que son fumadores describen a la mayoría de sus amigos como fumadores, al contrario de lo que describen los estudiantes que no lo son, que confirman que solo fuman la mitad o la minoría de sus amigos. Ello podría ser congruente con la visión que sostienen algunos trabajos de que el entorno familiar y el de los iguales influyen en el inicio del consumo de tabaco, atribuido al aprendizaje por imitación desde la infancia, así como al interés de experimentar algunas de las conductas realizadas por los adultos ${ }^{23-27}$.

Es relevante la distinta percepción que tienen sobre el tabaco las mujeres y los hombres en este estudio. Mientras que estos últimos creen, en mayor proporción que las mujeres, que facilita la relación grupal, existe un mayor porcentaje de mujeres que lo perciben como una ayuda para controlar el peso y una estrategia para sentirse bien. Estudios realizados en adolescentes han puesto de manifiesto aspectos similares en relación con el peso y con el manejo de las emociones negativas en las chicas ${ }^{12,28}$. Todo ello indica que la información destinada a los jóvenes debería incluir la perspectiva de género, para corregir la idea equivocada de los posibles beneficios que aportan los cigarrillos, sobre todo entre las mujeres ${ }^{23,29}$. Destacar que en esta muestra el porcentaje de mujeres fumadoras es superior al de hombres fumadores, sin existir diferencias, entre ambos, en el patrón de consumo (12 cig/día), en contra de lo comunicado hasta ahora en la mayoría de los estudios $^{3}$. Pensamos que este hallazgo refleja el avance a lo largo del estadio III del modelo evolutivo de la epidemia tabáquica ${ }^{30}$ en el que se encuentra nuestro país y confirma la tendencia de los últimos años del elevado consumo de tabaco por parte de las mujeres españolas.

En cuanto al porcentaje de fumadores, el 17,3\% fuma a diario y el $13,5 \%$ lo hace de manera ocasional. Aunque existe un acuerdo unánime en que el inicio del tabaquismo se produce en la adolescencia, algunos estudios han puesto de manifiesto que en la etapa universitaria se incorporan al consumo entre el $20 \%$ y el $30 \%$ de aquellos alumnos que se definen como fumadores a diario al finalizar la licenciatura ${ }^{31}$.

En los últimos años, en España se ha producido un cambio en los patrones y los estilos de diversión de los jóvenes ${ }^{32}$. Respecto al alcohol, según datos confirmados por el Observatorio Español sobre Drogas, el modelo de consumo de bebidas alcohólicas entre los jóvenes es frecuente los fines de semana y en días festivos, y para conseguir los efectos de embriaguez ${ }^{33}$, por ello quizás encontramos un número tan elevado de borracheras en los últimos 6 meses. En nuestro estudio, las mujeres refieren más el consumo de fin de semana y los hombres los días entre semana, generalmente, los dias de clase. Estas diferencias se podrian atribuir a una menor aceptación social del consumo de alcohol por parte de las mujeres.

Aun así la normalización existente sobre el consumo de alcohol no es extraña en nuestro país, ya que en muchas ocasiones el inicio del consumo esta ligado a la propia familia, en acontecimientos y/o fiestas señaladas debido al contexto cultural. Se debería revisar también el problema actual de permisividad de consumo de alcohol en la calle, así como la regulación y sanción de la venta a menores de edad $^{32}$.

Después del análisis de esta muestra de estudiantes, se podría pensar que el inicio tabáquico temprano puede actuar como la puerta de entrada para el consumo de alcohol y otras drogas no institucionalizadas ${ }^{34,35}$. En esta población, los estudiantes que son fumadores empezaron a beber alcohol a una edad más temprana y se emborrachan actualmente con mayor frecuencia.

En general el consumo de cualquier sustancia adictiva está relacionado, entre otros factores, con la percepción del riesgo y el acceso al producto. Así, se podría pensar también en nuestro estudio, que una mayor disponibilidad económica en los estudiantes que compatibilizan sus estudios con actividad laboral se asocia con el hecho de ser fumador, lo que por otra parte refuerza el potencial efecto preventivo del aumento del coste del producto recomendado por distintas instituciones de salud pública ${ }^{36}$. En cuanto a la percepción del riesgo, los jóvenes consideran que la enfermedad y la muerte son fenómenos muy lejanos en sus vidas por lo que no valoran los riesgos que conlleva el consumo de estas sustancias ${ }^{37}$. El alto nivel de tolerancia y permisividad hacia el consumo de tabaco y alcohol que ha habido en los últimos años en España contribuye a una menor percepción del riesgo que conlleva fumar y beber. Todo ello, junto a la falsa sensación de control que tienen al inicio, disminuye la percepción del riesgo que supone consumir estas sustancias, lo que favorece el consumo ${ }^{33}$.

Desde el punto de vista de salud pública, resulta muy preocupante el incremento del consumo de cannabis entre los jóvenes, al igual que sucede en la mayoría de los paises europeos ${ }^{35,38}$. Queda clara la relación entre tabaco y cannabis en esta muestra, ya que el $84 \%$ de los fumadores de tabaco a diario han probado los porros, y un porcentaje considerable de personas que son ex fumadoras continúan fumando porros en alguna ocasión. La comprensión de las implicaciones de una iniciación temprana en el consumo y las medidas que podrían resultar adecuadas es una de las complejas cuestiones para la salud pública y las políticas de control. 
Como limitaciones determinadas por el propio diseño del estudio, destacar las relacionadas con la elaboración del cuestionario y la tasa de respuesta obtenida. La población analizada no está enmarcada en la adolescencia, por ello los cuestionarios utilizados en escolares no recogían la información necesaria y hubo que adaptarlos a la muestra que se quería estudiar. En cuanto a la encuesta utilizada, a pesar de que existen otros cuestionarios para esta edad, algunos no hacian alusión al objetivo planteado, el consumo de tabaco principalmente, y otros eran muy extensos o muy centrados en una sola droga.

La mayoria de las encuestas en población universitaria se realizan en el aula por resultar el entorno más accesible, aunque se asume un cierto sesgo de selección que conlleva una infraestimación en los resultados, debido a que los alumnos que van a clase con regularidad son los que tienen hábitos de vida más saludables ${ }^{31}$. Por esta razón, se optó en este estudio por realizar la recogida de datos en el momento de la matrícula. En la Universidad de Zaragoza, al igual que en otras universidades, se ha incorporado desde hace unos años la matrícula por Internet. Este hecho ha contribuido a obtener una menor tasa de respuesta en este trabajo, que aunque es inferior a la obtenida en estudios que recogen datos en el aula, resulta superior a aquellos que han utilizado otras metodologías de comunicación como el correo postal| ${ }^{15,31}$. Todo ello sugiere la necesidad de utilizar en mayor medida las nuevas tecnologías en futuros estudios de investigación. Inicialmente se plantearon otras posibilidades, como la inclusión del cuestionario en la aplicación informática o la realización de un muestreo aleatorio, opciones que fueron denegadas por el Vicerrectorado de Estudiantes argumentando las limitaciones establecidas por la Ley de Protección de Datos, la complejidad del proceso y la ausencia de una base común de datos.

En cuanto a la sinceridad de los alumnos en las respuestas se valoró como poco comprometida, dado que la cumplimentación del cuestionario fue voluntaria y se realizaba en una zona diferente donde recogían la documentación, además de ser los propios alumnos los que lo introducian en un sobre cerrado anónimo. El hecho de que en esta muestra haya un porcentaje mayor de mujeres que inician estudios universitarios, refleja la misma situación que hay actualmente en las aulas de las demás universidades españolas.

Los resultados del estudio confirman la necesidad de dotar a los jóvenes de habilidades sociales que les ayuden a enfrentar determinadas situaciones de presión de grupo. En este ámbito, potenciar la práctica de ejercicio físico puede contribuir a prevenir el consumo de tabaco y otras drogas ${ }^{14}$, sin olvidar el papel que juegan los padres en la prevención del futuro consumo de sus hijos. A pesar de los avances realizados en nuestro país en las políticas de prevención del tabaquismo ${ }^{39}$, el consumo de drogas supone en la actualidad una asignatura pendiente que requiere importantes medidas de prevención que contribuyan a modificar la percepción del riesgo y la aceptación social, para lograr así la disminución del consumo entre los jóvenes.

\section{AGRADECIMIENTOS}

Para el desarrollo de este estudio se contó con el apoyo del Vicerrectorado de Estudiantes de la Universidad, y con la colaboración de las secretarías de las Facultades y Escuelas Universitarias de Zaragoza, Huesca y Teruel de la Universidad de Zaragoza.

\section{REFERENCIAS}

1. Ariza $C$, Nebot M, Villalbi JR, Diez E, Tomás Z, Valmayor S. Tendencias en el consumo de tabaco, alcohol y cannabis de los escolares de Barcelona (1987-1999). Gac Sanit 2003; 17: 190-5.

2. Ministerio de Sanidad y Consumo. Encuesta Nacional de Salud de 2006. Madrid, 2007

3. Ministerio del Interior. Delegación del Gobierno para el plan Nacional sobre Drogas. Secretaria General Técnica. Observatorio Español sobre Drogas. Informe 2004. Madrid, 2004.

4. Sánchez Pardo L. Consumo de alcohol en la población juvenil. Adicciones 2002; 14 Supl. 1: 99-113.

5. Nebot M, Tomás Z, Ariza C, Valmayor S, López MJ, Juárez 0. Factores asociados al inicio del tabaquismo: seguimiento a los 3 años de una cohorte de escolares. Arch Bronconeumol 2004; 40: 495-501.

6. Bandura A, Walters RH. Aprendizaje social y desarrollo de la personalidad. Madrid: Alianza Universidad; 1979.

7. Becoña E, Palomares A, Garcia MP. Tabaco y Salud. Madrid: Pirámide; 1994.

8. Froján MX, Santacreu J. Modelo de génesis, adquisición, mantenimiento e insatisfacción con el hábito de fumar. Boletín de Psicología 1992; 34: 7-25.

9. Jackson C. Cognitive susceptibility to Smoking and initiation of Smoking during childhood: a longitudinal study. Prev Med 1997; 27: 129-34.

10. Nebot $M$, Tomás Z, Ariza C, Valmayor S, Mudde A. Factores asociados con la intención de fumar y el inicio del hábito tabáquico en escolares: resultados del estudio ESFA en Barcelona. Gac Sanit 2002; 16: 131-8.

11. Ariza C, Nebot M. Predictores de la iniciación al consumo de tabaco en escolares de enseñanza secundaria de Barcelona y Lleida. Rev Esp Salud Pública 2002; 76: 227-38.

12. Nerín I, Beamonte $A$, Gargallo $P$, Jiménez-Muro $A$, Marqueta A. Ganancia ponderal al dejar de fumar y su relación con la ansiedad. Arch Bronconeumol 2007; 43: 9-15.

13. Nerín I, Crucelaegui A, Novella P, Ramón y Cajal P, Sobradiel $N$, Gericó R. Encuesta sobre tabaquismo en estudiantes universitarios en relación con la práctica de ejercicio físico. Arch Bronconeumol 2004; 40: 5-9.

14. Steptoe A, Wardle J, Cui W, Bellisle F, Zotti A, Baranyai R, et al. Trends in smoking, Diet, Physical Exercise and Attitudes toward Health in European University Students from 13 countries, 1990-2000. Prev Med 2002; 35: 97-104.

15. Guillén D, Nerín I, Mas A, Crucelaegui A. Estudio de la fiabilidad de una encuesta utilizada para valorar la prevalencia, los conocimientos y las actitudes sobre el tabaquismo en estudiantes de medicina. Arch Bronconeumol 2003; 39: 159-66. 
16. Garcia de Albéniz XA, Guerra-Gutiérrez F, Ortega-Martinez $R$, Sánchez-Villegas A, Martínez-González MA. Consumo de tabaco en titulados universitarios. El proyecto SUN (Seguimiento Universidad de Navarra). Gac Sanit 2004; 18: 108-17.

17. Ministerio del Interior. Observatorio Europeo de las Drogas y las Toxicomanias. Informe Anual 2007.

18. De Vries H, Mudde A, Kremers S, Wetzels J, Utiers E, Ariza C, et al. The European Smoking Prevention Framework Approach (ESFA): Short-term effects. Health Edu Res 2003; 18: 649-63.

19. De Vries H, Dijk F, Wetzels J, Mudde A, Kremers S, Ariza C, et al. The European Smoking Prevention Framework Approach (ESFA): effects after 24 and 30 months. Health Edu Res 2006; 21: 116-32.

20. Ariza $C$, Nebot $M$, Jané $M$, Tomas Z, De Vries H. El proyecto ESFA en Barcelona: un programa comunitario de prevención del tabaquismo en jóvenes. Prev Tab 2001; 3: 70-7.

21. Connelly J, Green J, Lechner L, Mittelmark MB, Rigby AS, Roberts C. The European Smoking Prevention Framework Approach (ESFA) project: observations of six commentators. Health Edu Res 2003; 18: 664-77.

22. Tyc VI, Hadley W, Allen D, Varnell S, Ey S, Rai SN et al. Predictors of smoking intentions and smoking status among nonsmoking and smoking adolescents. Addict Behav 2004; 29: 1143-7.

23. Villabí JR. Dejar de fumar durante el embarazo. Med Clin 2005; 124: 104-5.

24. Sánchez Agudo L. Tabaquismo en la infancia. Arch Bronconeumol 2004; 40: 1-4.

25. Leatherdale ST, Cameron R, Brown KS, Jolin MA, Kroeker C. The influence of friends, family, and older peers on smoking among elementary school students: Low-risk students in high-risk schools. Prev Med 2006; 42: 218-22.

26. Andersen MR, Leroux BG, Marek PM, Peterson AV, Kealey KA, Bricker J et al. Mothers' Attitudes and Concerns about Their Children Smoking: Do they Influence Kids? Prev Med 2002; 34: 198-206.
27. Font-Mayolas S, Planes M. Efectos del modelado por parte de la familia, pareja y amigos en la conducta de fumar de jóvenes universitarios. Adicciones 2000; 12: 467-77.

28. Nerín I, Jiménez-Muro A, Marqueta A. Perspectiva de género: Tabaco y Mujer. En: Barrueco M, Hernández MA, Torrecilla M, editores. Manual de prevención y tratamiento del tabaquismo. Madrid: GSK; 2006. p. 417-42.

29. Joossens L, Sasco A, Salvador T, Villalbi JR. Las mujeres y el tabaco en la Unión Europea. Rev Esp Salud Pública 1999; 73: 3-11.

30. López AD, Collishaw NE, Piha T. A descriptive model of the cigarette epidemic in developed countries. Tob Control 1994; 3: 242-7.

31. Mas $A$, Nerín I, Barrueco $M$, Cordero J, Guillén $D$, Jiménez-Ruiz $C_{1}$ et al. Consumo de tabaco en estudiantes de sexto curso de medicina de España. Arch Bronconeumol 2004; 40: 403-8.

32. Calafat $A$, Juan $M$, Becoña $E$, Castillo $A$, Fernández $C$, Franco $M$, et al. El consumo de alcohol en la lógica del botellón. Adicciones 2005; 17: 193-202.

33. Pascual F. Percepción del alcohol entre los jóvenes. Adicciones 2002; 14 Supl. 1: 123-31.

34. Nebot M, Giménez $E_{1}$ Ariza $C$, Tomás Z. Tendencias en el consumo de tabaco, alcohol y cannabis en los adolescentes de Barcelona entre 1987 y 2004. Med Clin 2006; 126: 157-9.

35. Kashdan TB, Vetter CJ, Collins RL. Substance use in young adults: Associations with personality and gender. Addict Behav 2005; 30: 259-69.

36. Martín E. Jóvenes y Alcohol. Adicciones 2002; 14: 135-7.

37. Guallar-Castillón P, Rodríguez F, Diez L, Banegas JR, Lafuente $P$, Del Rey J. Consumo de tabaco y salud subjetiva en España. Med Clin 2001; 116: 451-3.

38. Font-Mayolas S, Gras ME, Planes M. Análisis del patrón de consumo de cannabis en estudiantes universitarios. Adicciones 2006; 18: 337-44.

39. Ley 28/2005, de 26 de Diciembre, de Medidas Sanitarias frente al tabaquismo y reguladora de la venta, el suministro, el consumo y la publicidad de los productos de tabaco. 